\title{
A new solver for granular avalanche simulation: Indoor experiment verification and field scale case study
}

\author{
XiaoLiang Wang ${ }^{1}$, and $\mathrm{JiaChun} \mathrm{Li}^{1,2^{*}}$ \\ ${ }^{1}$ Key Laboratory for Mechanics in Fluid Solid Coupling Systems, Institute of Mechanics, Chinese Academy of Sciences, Beijing 100190, China; \\ ${ }^{2}$ School of Engineering Science, University of Chinese Academy of Sciences, Beijing 100049, China
}

Received July 5, 2017; accepted August 22, 2017; published online October 27, 2017

\begin{abstract}
A new solver based on the high-resolution scheme with novel treatments of source terms and interface capture for the Savage-Hutter model is developed to simulate granular avalanche flows. The capability to simulate flow spread and deposit processes is verified through indoor experiments of a two-dimensional granular avalanche. Parameter studies show that reduction in bed friction enhances runout efficiency, and that lower earth pressure restraints enlarge the deposit spread. The April 9, 2000, Yigong avalanche in Tibet, China, is simulated as a case study by this new solver. The predicted results, including evolution process, deposit spread, and hazard impacts, generally agree with site observations. It is concluded that the new solver for the Savage-Hutter equation provides a comprehensive software platform for granular avalanche simulation at both experimental and field scales. In particular, the solver can be a valuable tool for providing necessary information for hazard forecasts, disaster mitigation, and countermeasure decisions in mountainous areas.
\end{abstract}

avalanche, landslide, WAF, TVD

PACS number(s): 45.70.Ht, 47.11.Df, 47.57.Gc, 92.40.Ha

Citation: X. L. Wang, and J. C. Li, A new solver for granular avalanche simulation: Indoor experiment verification and field scale case study, Sci. China-Phys. Mech. Astron. 60, 124712 (2017), doi: 10.1007/s11433-017-9093-y

\section{Introduction}

Large scale mass movements such as landslides, avalanches, and debris flows are common in mountainous regions worldwide. Usually triggered by earthquake or heavy rainfall, they can threaten critical infrastructure such as dams and power stations, and can even threaten human life. At least 12000 landslides from a total of 40000-50000 during the Wenchuan earthquake in 2008 caused damage [1]. The Daguangbao landslide, which carried approximately $750 \times 10^{6}$ $\mathrm{m}^{3}$ of granular material, was the world's largest. The April

*Corresponding author (email: jcli05@imech.ac.cn)
9, 2000, Yigong avalanche in Tibet, China, transported up to $300 \times 10^{6} \mathrm{~m}^{3}$ of granular material and formed a natural deposit dam $10 \mathrm{~km}$ away [2,3]. Furthermore, granular avalanche deposits in gullies with ample loose sediment supply may increase the solid fraction in the runoff water and thus cause debris flow. Granular avalanches can also build up high natural deposit dams, which are then often prone to dam break from piping or overtopping from the avalanche lake formed behind. Hence, it is very important to have a credible software tool available to predict the dynamic processes of granular avalanches and assess their direct effects and possible secondary impacts such as debris flow or dam break flood.

Granular avalanches are made up of cohesionless particles 
of different shapes and sizes. With various particle cluster structures, granular material may exhibit behaviors in different physical regimes dependent on the input energy, such as granular solids, liquids, or gases [4]. However, the lack in scientific knowledge of its constitutive law has led to poor granular avalanche modeling $[5,6]$. The physical process of granular avalanches has been treated by some researchers as a large deformation problem [7], and as a flow problem by others [8-10]. In addition, bed erosion entrainment can considerably enhance the volume and energy of granular avalanches. For example, the Tingshan landslide [11] transported a total material volume of $20000 \mathrm{~m}^{3}$, yet its initial unstable sliding block was only about $400 \mathrm{~m}^{3}$. The physical laws and mechanisms in granular materials and flow add difficulty and complexity to this challenging issue.

The thickness of a granular avalanche is usually much less than its horizontal extent scale. Assuming vertical hydrostatic pressure like in shallow water problems, most granular avalanche models are derived by vertically integrating the continuity and momentum equations from bed to free surface. In particular, the 1989 Savage-Hutter (SH) model [12] for snow avalanches was the first to consider two very significant factors for granular materials behavior: earth pressure and Coulomb friction. The SH model's capacity for simulating both transport and deposit of granular materials for many geophysical mass flows led to a break-through in granular avalanche modeling and laid a solid foundation for other geophysical mass flows, such as snow avalanches, debris flows, and volcanic lava. Since then, a number of advances have been made. Pudasaini and Hutter [13] added geographic effects, including gully curvature and twist, into the SH model. Iverson and Denlinger [9] developed the famous Coulomb mixture model, with pore water pressure explicitly considered. The advanced mixture model with pore water pressure evolution equation and critical state behavior considered was proposed in 2014 [14]. Pitman and Le [15] and Pudasaini [16] established two phase depth-averaged equations for debris flow, first applied to study water-soil mixture flow in air (such as debris flow), and later applied to submarine landslide motion to assess the stability of pipe system foundations [17]. In summary, two directions have emerged for granular avalanche model development since the 1989 SH model. On one hand, more accurate physical constitutive laws for granular material were considered, based either on phenomenological experiments [18] or on discrete element simulations $[19,20]$. In particular, the shear rate-dependent $\mu(I)$ model of Gray and Edwards [21] for the flowing granular material was suggested and incorporated into the SH model. This development turned out very helpful for elucidating fascinating physical phenomena such as size segregation [22] and roll waves [23], among others. On the other hand, physics-based entrainment laws for bed erosion were examined, such as the Hungr model [24] and Iverson's jump entrainment law [25].
These new advances are pivotal for simulating granular flow in realistic natural environments.

As for numerical approach, Savage and Hutter [26] were the first to use algorithms based on the Lagrangian viewpoint, and modeled a steep profile via the artificial dissipation method. Based on the SH model's hyperbolic characteristics, later numerical methods borrowed many concepts from computational gas dynamics, such as Godunov type schemes [27], in order to capture discontinuities. Pitman et al. [28] numerically solved one-dimensional (1D) and two-dimensional (2D) $\mathrm{SH}$ models with the monotonic upstream scheme for conservation laws (MUSCL) method to reconstruct the left and right Riemann invariants. They used Harten, Lax and van Leer (HLL) and HLL-contact (HLLC) approximate Riemann solvers for the Riemann problem with the Davis approximation to estimate left and right wave speeds [29]. Similarly, Zhai et al. [30] solved the 2D SH model and applied their numerical schemes to numerous experimental scale problems. Pudasaini et al. [8] constructed the non-oscillatory central differencing (NOC) schemes for a mixture model with pore water pressure considered, then successfully applied for dry and wet granular flow. While Denlinger and Iverson [31] developed the HLLC Riemann solver for their Coulomb mixture model in 2001, the Roe Riemann solver for their advanced mixture model was built in 2014 [32]. Both were applied in a large outdoor flume experiment for debris flows. The two phase Pitman model exhibits some non-conservative behaviors and mixed-type mathematical characteristics. Pelanti et al. [33,34] developed a relaxation technique and a Roetype scheme for the reconstructed Pitman model equation. While Pudasaini [16] still used the NOC scheme for the two phase equation with several 1D problems on slopes, Greco et al. [35] and He et al. [36] similarly solved the mixedtype governing equations of two phase granular flow by using the Roe-like Riemann solver. Note that the high-resolution essentially non-oscillatory (ENO) and weighted-essentially non-oscillatory (WENO) schemes [37] in gas dynamics have recently found application in shallow water problems but not yet in granular avalanche flow problems. In addition, mesh-free methods such as smoothed particle hydrodynamics (SPH) [10] and material point method (MPM) [38] have now become another potential approach for granular flow problems. In summary, both theoretical and numerical schemes in granular avalanche modeling are still in an immature stage. The accuracy of granular avalanche simulation heavily depends on understanding of constitutive law, size segregation phenomena, and entrainment mechanisms, among others. Although numerical algorithms such as the Godunov scheme have found wide applications in granular flow study, there remain deficiencies in dealing with new constitutive law, discontinuity capture, erosion and entrainment modeling, and wet/dry front treatment in the same computational framework. Furthermore, today's numerical models 
show ability in simulating of well-controlled granular flows, but not yet in predicting actual granular avalanches in mountainous regions. Thus, in this study a new solver based on the Godunov-type method is developed to simulate both indoor and field scale granular avalanches.

This article is arranged as follows. In sect. 2, the novel solver, including its mathematical equation, numerical method, and parameter discussion is presented. In sect. 3 , indoor experiments are discussed for solver verification which includes comparison of computations and measurements. Then in sect. 4, a case study using the new solver is presented: the simulation of the April 9, 2000, Yigong avalanche in Tibet, China. Finally, concluding remarks are presented.

\section{Mathematical formulation, method and parametric study}

\subsection{Mathematical formulation}

\subsubsection{Governing equation}

Based on the hydrostatic pressure assumption, the $1989 \mathrm{SH}$ model [12] was first derived by integrating the full equation from bed to free surface for the study of granular avalanches. While the relationship between lateral stress and vertical stress is established by using the earth pressure theorem, the bed resistance was required to obey the Coulomb friction law. The resultant compact conservative equation reads as:

$\frac{\partial \boldsymbol{U}}{\partial t}+\frac{\partial \boldsymbol{F}}{\partial x}+\frac{\partial \boldsymbol{G}}{\partial y}=\boldsymbol{S}$,

where

$\boldsymbol{U}=(h, h u, h v)^{\mathrm{T}}$,

$\boldsymbol{F}=\left(h u, h u^{2}+\frac{1}{2} k_{x} g_{z} h^{2}, h u v\right)^{\mathrm{T}}$,

$\boldsymbol{G}=\left(h v, h u v, h v^{2}+\frac{1}{2} k_{y} g_{z} h^{2}\right)^{\mathrm{T}}$.

The source terms are

$$
\begin{gathered}
\boldsymbol{S}=h\left(0, g_{x}-\frac{u}{\sqrt{u^{2}+v^{2}}} \tan \delta\left(g_{z}+\frac{u^{2}}{r_{x}}\right)-g_{z} \frac{\partial z_{\mathrm{b}}}{\partial x},\right. \\
\left.g_{y}-\frac{v}{\sqrt{u^{2}+v^{2}}} \tan \delta\left(g_{z}+\frac{v^{2}}{r_{y}}\right)-g_{z} \frac{\partial z_{\mathrm{b}}}{\partial y}\right)^{T},
\end{gathered}
$$

where $h, u$, and $v$ denote flow depth and the depth-averaged velocity components in two horizontal directions of the granular avalanche. $\boldsymbol{g}=\left(g_{x}, g_{y}, g_{z}\right)^{\mathrm{T}}$ are the gravity vector components. The terms in the source term represent gravity, bed friction, centrifugal force, and bed variation effects respectively, where $z_{\mathrm{b}}$ is the bed elevation, and $r_{x}, r_{y}$ and $k_{x}, k_{y}$ are the curvature radii and the earth pressure coefficients in streamwise and lateral directions, as shown in eqs. (2) and (3).

$k_{x}=2\left(1 \mp \sqrt{1-\cos ^{2} \phi\left(1+\tan ^{2} \delta\right)}\right) \sec ^{2} \phi-1$,

$k_{y}=\frac{1}{2}\left(1+k_{x} \mp \sqrt{\left(1-k_{x}\right)^{2}+4 \tan ^{2} \delta}\right)$,

where $\phi$ is the internal friction angle, and $\delta$ is the bed friction angle.

For positive velocity gradients, the active ("-") earth pressure coefficient is chosen, while the passive ("+") earth pressure coefficient is used otherwise.

\subsubsection{Remarks on SH model}

In the $\mathrm{SH}$ model, there are two constitutive characteristics of granular material available which differ from those of pure fluids, namely, anisotropic stresses and friction behavior. An earth pressure coefficient either larger or smaller than unit governs the lateral constraint and final deposit distribution, while the bed friction governs the stagnation and runout distance of granular material. However, pure fluids with a unit pressure coefficient always keep running in a region without boundary. In regard to flowing granular material, also termed as granular liquids, it is controversial whether these two assumptions are valid $[17,39,40]$. For instance, Gray and Edwards [21], Jop et al. [39], and Forterre and Pouliquen [41] experimentally proposed the $\mu(I)$ rate-dependent friction law based on a phenomenological model. Others have assumed the earth pressure coefficient as unit, like in the shallow water equation $[21,42]$. Essentially, the two friction parameters in the $\mathrm{SH}$ model mainly govern streamwise/lateral spread and runout distance, somewhat different from their role in geomechanics.

\subsection{Numerical method}

\subsubsection{WAF-TVD scheme}

Structured Cartesian mesh is used to discretize the whole domain. A weighted average flux (WAF) scheme incorporating the total variation diminishing (TVD) technique is borrowed [27] to manage the advection term in eq. (1) for a structured Cartesian mesh. Eq. (1) is thus simplified as:

$\frac{\partial \boldsymbol{U}}{\partial t}+\boldsymbol{A} \frac{\partial \boldsymbol{U}}{\partial x}+\boldsymbol{B} \frac{\partial \boldsymbol{U}}{\partial y}=\boldsymbol{S}(\boldsymbol{U})$

where

$$
\begin{aligned}
& \boldsymbol{A}=\frac{\partial \boldsymbol{F}}{\partial \boldsymbol{U}}=\left[\begin{array}{lll}
0 & 1 & 0 \\
-u^{2}+k_{x} g_{z} h & 2 u & 0 \\
-u v & v & u
\end{array}\right], \\
& \boldsymbol{B}=\frac{\partial \boldsymbol{G}}{\partial \boldsymbol{U}}=\left[\begin{array}{lll}
0 & 0 & 1 \\
-u v & v & u \\
-v^{2}+k_{y} g_{z} h & 0 & 2 v
\end{array}\right] .
\end{aligned}
$$


If we define the cell averaged value as $U_{i, j}=\frac{1}{\Delta x \Delta y} \int U \mathrm{~d} x \mathrm{~d} y$, then the Godunov scheme reads as:

$$
\begin{aligned}
U_{i, j}^{(n+1)}= & U_{i, j}^{n}-\frac{\Delta t}{\Delta x}\left(F_{i+\frac{1}{2}, j}-F_{i-\frac{1}{2}, j}\right) \\
& -\frac{\Delta t}{\Delta y}\left(G_{i, j+\frac{1}{2}}-G_{i, j-\frac{1}{2}}\right),
\end{aligned}
$$

where $F_{i+\frac{1}{2}, j}$ and $G_{i, j+\frac{1}{2}}$ are numerical inter-cell fluxes, calculated via the WAF scheme to achieve a second-order accuracy as shown in eqs. (6) and (7). Following a first-order Godunov prediction, a second-order WAF method is used to construct the numerical flux.

$F_{i+\frac{1}{2}, j}=L_{x, \Delta t 2}^{\mathrm{waf}}\left(L_{y, \Delta t 2}^{\mathrm{god}}\left(U_{i, j}^{k}\right), L_{y, \Delta t / 2}^{\mathrm{god}}\left(U_{i+1, j}^{k}\right)\right)$,

$G_{i, j+\frac{1}{2}}=L_{y, \Delta t 2}^{\mathrm{waf}}\left(L_{x, \Delta t 2}^{\mathrm{god}}\left(U_{i, j}^{k}\right), L_{x, \Delta t / 2}^{\mathrm{god}}\left(U_{i, j+1}^{k}\right)\right)$,

where $L_{x, \Delta t / 2}^{\mathrm{god}}$ and $L_{y, \Delta t / 2}^{\mathrm{god}}$ are first-order Godunov operators:

$L_{s, \Delta t}^{\mathrm{god}}\left(U^{k}\right)=U^{k}+\frac{\Delta t}{\Delta s}\left[K_{l-\frac{1}{2}}^{\mathrm{god}}-K_{l+\frac{1}{2}}^{\mathrm{god}}\right]$,

where $s$ may denote $x$ or $y$, and $K_{l+\frac{1}{2}}^{\text {god }}$ is the first-order Godunov upwind flux.

The operator $L_{x, \Delta t / 2}^{\text {waf }}$ denotes the WAF operator:

$L_{s, \Delta t / 2}^{\text {waf }}\left(U_{\mathrm{L}}, U_{\mathrm{R}}\right)=\frac{1}{2}\left(E_{\mathrm{L}}+E_{\mathrm{R}}\right)-\frac{1}{2} \sum_{m=1}^{N} \operatorname{sign}\left(c_{m}\right) A_{m} \Delta E_{m}$.

Similarly, $s$ may denote $x$ or $y, E_{\mathrm{L}}$ and $E_{\mathrm{R}}$ are left and right fluxes, $c_{m}$ is the Courant number for the $m$ th wave in the Riemann solution, $\Delta E_{m}$ is the flux jump across the $m$ th wave, and $A_{m}$ is a limiter function for the $m$ th wave to limit the spurious oscillation which may happen near shocks.

The Riemann solution is calculated via the HLLC approximated Riemann solver as:

$F^{\mathrm{HLLC}}\left(U_{\mathrm{L}}, U_{\mathrm{R}}\right)=\left\{\begin{array}{cc}F\left(U_{\mathrm{L}}\right), & s_{\mathrm{L}} \geq 0, \\ F\left(U_{\mathrm{L}}\right)+s_{\mathrm{L}}\left(U_{\mathrm{L}}^{*}-U_{\mathrm{L}}\right), & s_{\mathrm{L}} \leq 0 \leq s_{*}, \\ F\left(U_{\mathrm{R}}\right)+s_{\mathrm{R}}\left(U_{\mathrm{R}}^{*}-U_{\mathrm{R}}\right), & s_{*} \leq 0 \leq s_{\mathrm{R}}, \\ F\left(U_{\mathrm{R}}\right), & s_{\mathrm{R}} \leq 0 .\end{array}\right.$

Further detail of the WAF method and the HLLC Riemann solver can be found in Toro's monograph [27].

The Davis estimate [28] is chosen for the left, middle and right wave speed as $s_{\mathrm{L}}=\min \left(u_{\mathrm{L}}-a_{\mathrm{L}}, u_{\mathrm{R}}-a_{\mathrm{R}}\right)$, $s_{*}=\frac{s_{\mathrm{L}} h_{\mathrm{R}}\left(u_{\mathrm{R}}-s_{\mathrm{R}}\right)-s_{\mathrm{R}} h_{\mathrm{L}}\left(u_{\mathrm{L}}-s_{\mathrm{L}}\right)}{h_{\mathrm{R}}\left(u_{\mathrm{R}}-s_{\mathrm{R}}\right)-h_{\mathrm{L}}\left(u_{\mathrm{L}}-s_{\mathrm{L}}\right)}$ and $s_{\mathrm{R}}=\max \left(u_{\mathrm{L}}+a_{\mathrm{L}}, u_{\mathrm{R}}+a_{\mathrm{R}}\right)$.

This Godunov-type method is stable with the CFL condition satisfied.

$\mathrm{CFL}=\max \left(\left|a_{x}\right|_{\max } \frac{\Delta t}{\Delta x},\left|a_{y}\right|_{\max } \frac{\Delta t}{\Delta y}\right) \leq \frac{1}{2}$.

For CFL number of 0.2 , the computations are found to be stable for all cases in this study.

\subsubsection{Splitting step integration}

Granular flows can adjust themselves when deposit takes place on variable mild slopes since the friction mechanisms differ from those in the shallow water problem. It is implied that a numerical scheme does not need to obey the C-property, considerably important in the shallow water problem [43]. Thus, the Strang splitting step method is used to handle the source term here:

$U_{i, j}^{n+1}=L\left(\frac{1}{2} \Delta t\right) \prod_{k=1}^{m} S\left(\frac{1}{m} \Delta t\right) L\left(\frac{1}{2} \Delta t\right)$.

To realize the Strang splitting step calculation, a half time step is first implemented to march forward the advection operator (termed as $L$ ), then integration of the source term (termed as $S$ ) is followed for sub-time steps, and finally another half time step for the advection term is implemented once again. In all of the numerical examples in this study, the computation is stable with one time step for the source term when the CFL number is 0.2 .

\subsubsection{Interface treatment}

The interface between material and open region must be treated properly. Recent decades have seen rapid progress in the thin layer method for this issue, including Bradford and Sanders [44], Iverson and Denlinger [9], and Zhai et al. [30] for hydraulic, debris, and granular flows, respectively. The depth and velocity calculations in the thin layer method should be suitably designed to render the above algorithm accurate and stable.

The interface treatment in this study is similar to the method shown in refs. [27,30]. For instance, when facing positive $x$ direction, a rarefaction wave transports towards the left if the right cell has zero depth. Using the Riemann invariant for the interface, we can obtain the propagating speed as $s=u_{\mathrm{L}}+2 a_{\mathrm{L}}$ to replace the estimated right-going wave speed in the HLLC Riemann solver.

Instead of a complicated interface location technique, we prefer to propose a novel modified thin layer method $[30,44,45]$ to overcome dry bed difficulty. First, we define a minimum depth $h_{\min }$ in almost dry region. For cell depth less than $h_{\text {min }}$, there are two possible circumstances. When the depth of all the neighboring cells is smaller than $h_{\min }$, we assume flow stops there. Otherwise, when some neighboring cells hold a depth larger than $h_{\min }$, the mass conservation equation is calculated without outflow and the velocity is extrapolated from the deepest neighboring cell. In this way, we can avoid nonphysical results such as negative depth cell, which happens in the method given by Zhai et al. [30] if both inflow and outflow are calculated for the mass conservation equation. We found that this treatment ensures computational stability until $h_{\min }$ is $10^{-5}$. 


\subsection{Parametric study}

The SH model contains two physical parameters: bed friction angle and internal friction angle. A series of cases are designed to examine the effects of both internal friction and bed friction angle on runout behavior.

An inclined plane is connected to an open horizontal runout surface with a transition arc zone in between as shown in Figure 1. The plane size is $25 \mathrm{~m} \times 40 \mathrm{~m}$ with $45^{\circ}$ inclination angle. The transition zone lies between 25 and $30 \mathrm{~m}$ (shown by the red dashed line), while the remaining part is the runout zone. A hemisphere-shaped granular mass with radius $2.5 \mathrm{~m}$ is heaped at $(7.5 \mathrm{~m}, 20 \mathrm{~m}$ ) (shown as a contour line in Figure 1) to model the unstable landslide.

We first assume a $35^{\circ}$ internal friction angle. Bed friction angles of $10^{\circ}, 15^{\circ}, 20^{\circ}, 25^{\circ}, 30^{\circ}$, and $35^{\circ}$ are chosen to study runout behavior.

Figure 2 plots deposition pattern at the center line ( $Y=$ $20 \mathrm{~m}$ ) for all the cases. There is clearly evident growth of runout distance with the reduction in bed friction angle. In

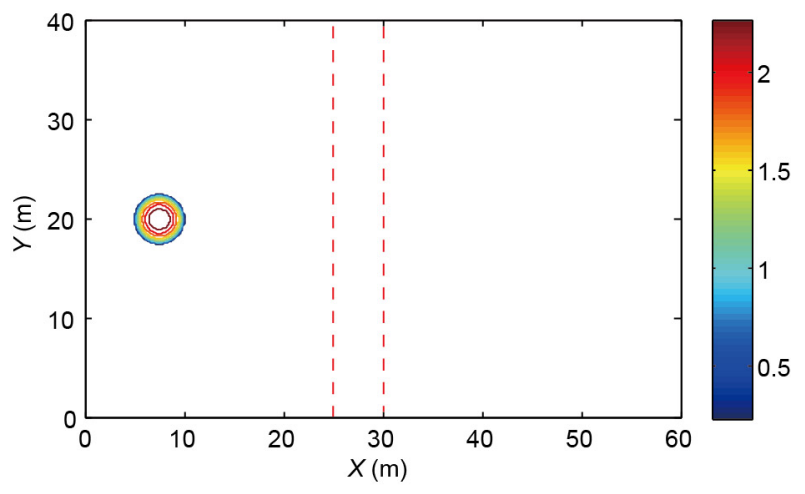

Figure 1 (Color online) Schematic diagram for runout tests. Contour lines denote the initial position where unstable granular materials are placed. The region between dashed lines is the transition zone, while the regions at the left and right are the inclined plane of $45^{\circ}$ and the runout zone, respectively.

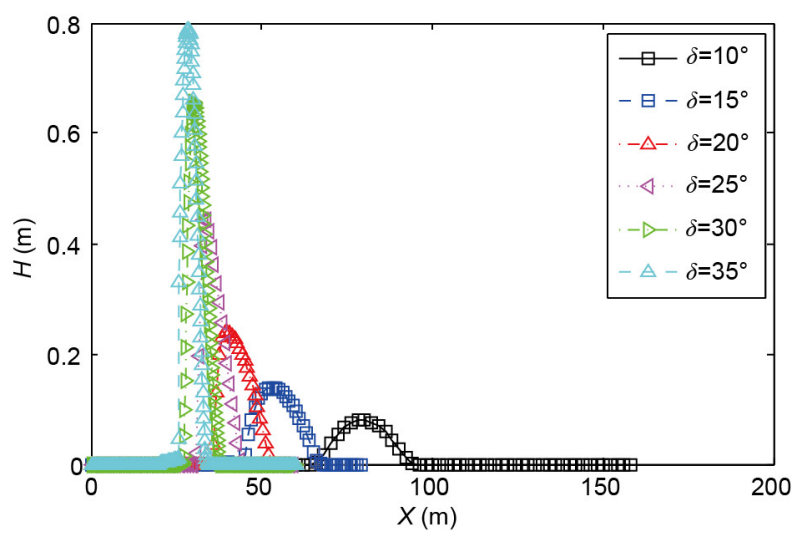

Figure 2 (Color online) Final deposition pattern at the center line for different bed frictions $\left(10^{\circ}, 15^{\circ}, 20^{\circ}, 25^{\circ}, 30^{\circ}\right.$ and $\left.35^{\circ}\right)$, with an internal friction angle of $35^{\circ}$. addition, the spread of landslides with a smaller bed friction angle extend wider, with a lower final maximum height.

Secondly, we assume the bed friction angle as $20^{\circ}$. Then, internal friction angles of $20^{\circ}, 25^{\circ}, 30^{\circ}$, and $35^{\circ}$ are chosen to analyze runout and deposition behaviors. Figure 3 shows deposit patterns at the center line $(Y=20 \mathrm{~m})$. Averaged runout distances with the same bed friction angle but different internal friction angles are found to be almost the same, however, the deposit varies greatly. Larger internal friction causes a wider spread with a lower maximum height in the streamwise direction.

The final deposit is a stagnation picture of the flowing granular materials on the runout zone, governed by the lateral constraint. The earth pressure in the main body of granular flow is in the active regime, while those in the front and tail regions are in the passive regime on the slope. Figure 4 shows the active and passive earth pressure coefficients as a function of bed friction angle or internal friction angle. The passive earth pressure coefficient increases with the internal friction angle, and is always larger than 1.0, while the active earth pressure coefficient decreases with internal friction value, and can be as small as about 0.5 . The smaller active earth pressure constraint in the lateral direction causes a wider deposit extension, as in Figure 3. The wider extension behavior in Figure 2 can also be understood by the same mechanism from Figure 4(a).

Runout efficiency (RE), defined as the ratio of runout distance to the initial height, is an important quantity in the assessment of avalanche hazards [25]. With two other series of cases for inclination angles of $30^{\circ}$ and $60^{\circ}$ computed, the relationship between $\mathrm{RE}$ and bed friction angle obtained is shown in Figure 5. We find that RE increases with the reduction of bed friction. For rough bed friction larger than $15^{\circ}$, RE never exceeds 2-3, implying that landslide stops right at or near the toe of a slope. For smooth bed friction cases, RE can be greater than 3 , meaning that the landslide may travel a

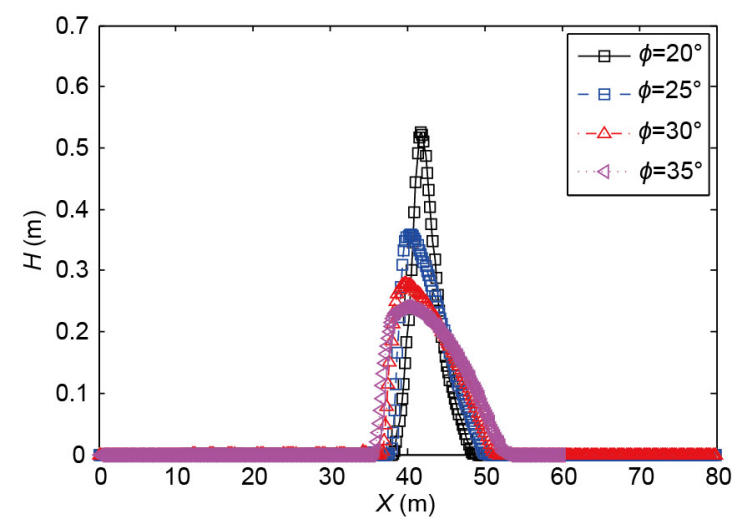

Figure 3 (Color online) Final deposit height at the center line for different internal friction angles $\left(20^{\circ}, 25^{\circ}, 30^{\circ}\right.$, and $\left.35^{\circ}\right)$ with bed friction angle of $20^{\circ}$. 

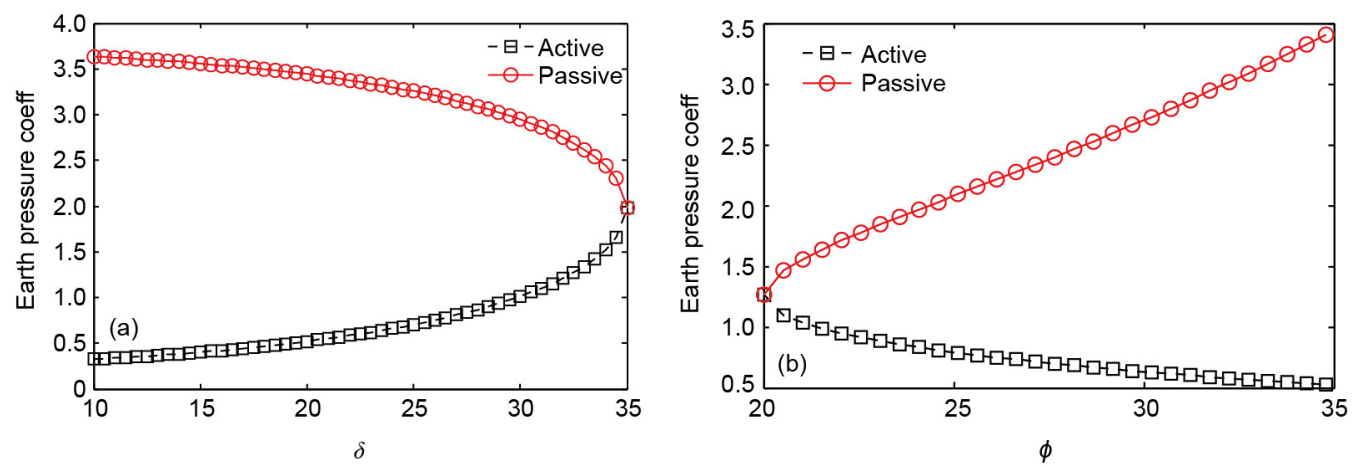

Figure 4 (Color online) Active and passive earth pressure coefficients as a function of bed friction with internal friction of $35^{\circ}$ (a), and as a function of internal friction with bed friction angle of $20^{\circ}(\mathrm{b})$.

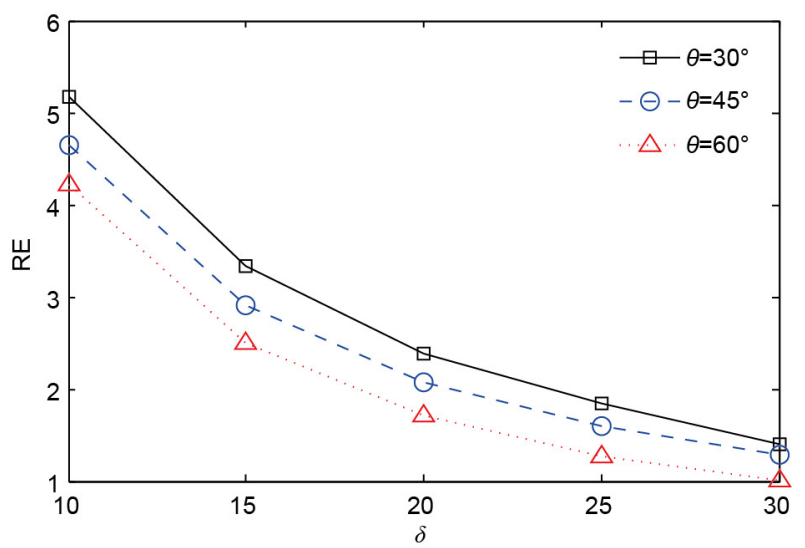

Figure 5 (Color online) Runout efficiency (RE) versus bed friction angle for different slopes with angles $30^{\circ}, 45^{\circ}$, and $60^{\circ}$. RE is defined as the ratio of runout distance to initial failure height.

long way prior to stopping. For giant landslides, avalanches or debris flows with volumes of larger than $10^{6} \mathrm{~m}^{3}$ either with or without water, RE can be as large as 10 according to the records $[9,46]$. The mechanism of giant long runout phenomena, such as acoustic fluidization by Melosh [46], air lubrication by Shreve [47] or soil liquefaction by Iverson and LaHusen [48] is still under discussion. However, it can be concluded that simulation is possible simply by setting a low equivalent bed friction angle in the SH model. However, further study of long runout behavior is necessary.

\section{Indoor experiment verification}

The new solver was first tested by 1D dam break and granular avalanche interaction simulations in ref. [49].

For further verification, we prefer to compare the simulation of two-dimensional granular avalanches with experiments performed on a planar slope with runout zone by Koch et al. [50] for two types of granular material. The planar slope with inclination $45^{\circ}$ was $3 \mathrm{~m}$ long and $2 \mathrm{~m}$ wide with a strip-type transition zone between 1.75 and $2.15 \mathrm{~m}$, while the runout zone was entirely horizontal. At the center line of the above section, a hemisphere cap with a radius $18.5 \mathrm{~cm}$ was used as the initial unstable granular material. The avalanche processes were recorded by high speed camera.

The No. 1 experiment with marmor granule about $5 \mathrm{~mm}$ in diameter is numerically simulated with parameters $\phi=42^{\circ}$ and $\delta=39^{\circ}$ from tilting experiments. Figure 6 shows the numerical results at six typical moments. The experiment results for the boundary zone are also shown in Figure 6, with circles denoting cells filled with at least one layer of particles and triangles denoting cells filled with particles only in a half. The unstable granular block in the hemisphere quickly rushed out and then slowed down in the runout zone. Figure 6 shows the formation of a "shock wave" when granular materials at the rear catch up with those in front in the runout zone-very similar to what happens in gas dynamics. In general, the simulated granular avalanche processes coincide well with the experiments. More specifically, numerical results for the front and tail seemed to be better than those for lateral spread on the slope. In the transition and runout zones, the simulated front of granular flow is well-positioned, while the simulated tail and lateral spread show some deviation from the experiments. Furthermore, the circles are found to better agree with the simulated front and tail boundary. In contrast, triangles representing granular flows only with collision as the main particle interaction mechanism are beyond the continuum regime of SH theory.

In summary, the new solver can simulate fundamental behavior of flow and deposit of granular avalanches as compared with indoor experiments, including elapse time, deposit depth, and extent. However, lateral spread prediction is not as satisfactory as longitudinal, implying that the earth pressure theory in soil mechanics may need further revision for granular material flow regimes.

\section{Case study: Yigong's field scale avalanche}

On April 9, 2000, a giant, fast, long runout avalanche swept through Yigong County in Tibet, China. A weathered deposit of rock debris as high as $3000 \mathrm{~m}$ above the Yigong River suddenly collapsed and rushed down a gully, eroding the bed 



Figure 6 (Color online) Depth evolution of No. 1 granular avalanche of Koch's experiment: numerical and experimental comparison. Depth contour is depicted by color, with bottom value as the particle diameter $(0.005 \mathrm{~m})$. The region between the red dashed lines is the transition zone. The moments of (a)-( $\mathrm{f}$ ) are $0.6 \mathrm{~s}, 0.84 \mathrm{~s}, 1.04 \mathrm{~s}, 1.23 \mathrm{~s}, 1.62 \mathrm{~s}$, and $2.46 \mathrm{~s}$, respectively.

and side banks. Finally, granular material blocked the Yigong River and formed a deposit dam of $4.6 \mathrm{~km}$ long, $3 \mathrm{~km}$ wide and $60 \mathrm{~m}$ thick on average with a total bulk volume about 3.8 $\times 10^{8} \mathrm{~m}^{3}$. A dam break happened nearly two months later, raising the water surface $55 \mathrm{~m}$ in Yigong Lake [2,3,51].

The elevation data of NASA's global digital elevation model (GDEM) based on advanced spaceborne thermal emission and reflection radiometer (ASTER) is used in this study (as shown in Figure 7(a)), which is deemed sufficiently detailed information for this particular landslide and deposit. Because no erosion is taken into account in the SH model, the total volume can only be roughly estimated at the failure position where the unstable landslide happened. It is very likely that the large runout distance of giant avalanches with volumes larger than $10^{6} \mathrm{~m}^{3}$ can be attributed to low bed friction, only one of the possible mechanisms among others (such as acoustic fluidization by Melosh [46], air lubrication by Shreve [47], or soil liquefaction by Iverson and LaHusen [48]). Up to now, the bed friction value for a giant avalanche has typically been obtained through experience or calibration. Lucas et al. [52] made statistical examinations on many long runout giant avalanches, landslides, and debris flows, and summarized a volume-dependent friction law for giant landslide motion. According to Lucas, the $3.8 \times 10^{8} \mathrm{~m}^{3}$ avalanche should be governed by an equivalent friction angle of about $11.3^{\circ}$. By considering this theory, several simulations with bed friction angles of $5^{\circ}, 10^{\circ}, 15^{\circ}$, and $20^{\circ}$ were chosen for calibration against the runout distance. Final depositions of Yigong's avalanche are shown in Figure 8. By comparing the simulated results of deposit spread and depth with the observations, a suitable bed friction angle of $\delta=12.5^{\circ}$ and an internal friction angle of $\phi=25^{\circ}$ were found for the Yigong avalanche.

With the selected parameters, the full transport and deposit processes are simulated numerically using a mesh of $400 \times 1000$, with depth evolution at 4 monitoring positions (Pos 1, Pos 2, Pos 3 and Pos 4) in Yigong River, as shown in Figure 7(b). Figure 9 plots snapshots of the Yigong landslide. After the initial slide on the hill, the slide accelerates very quickly along the gully, and collides with the gorge bank. Then it slows down on the runout zone, maintains momentum, and finally deposits across the Yigong River. The Yigong River is almost fully blocked by loose soil deposited along the gully upward until the narrow gorge. The temporal 

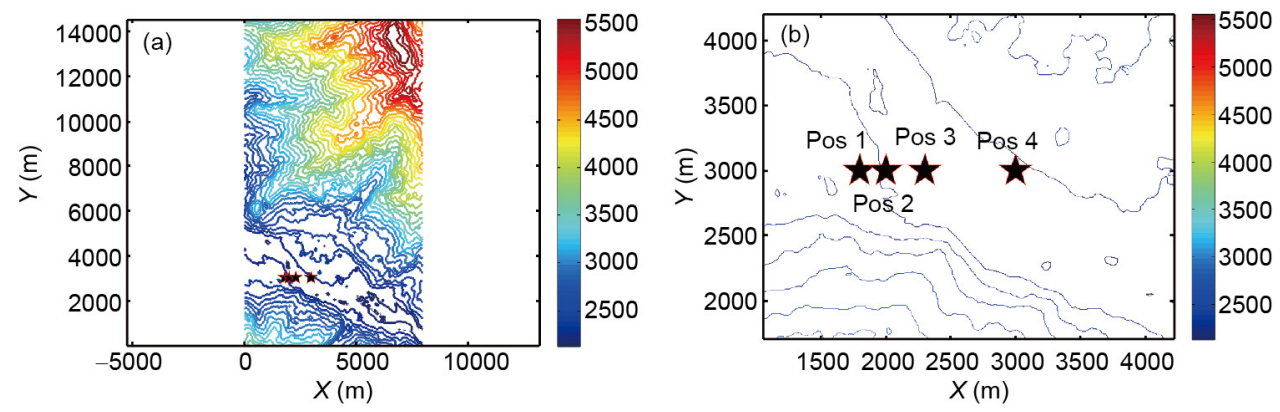

Figure 7 (Color online) Reconstructed computational topographic map based on ASTER GDEM elevation data (a), and 4 monitoring positions of Yigong avalanches are indicated by star symbols (b).
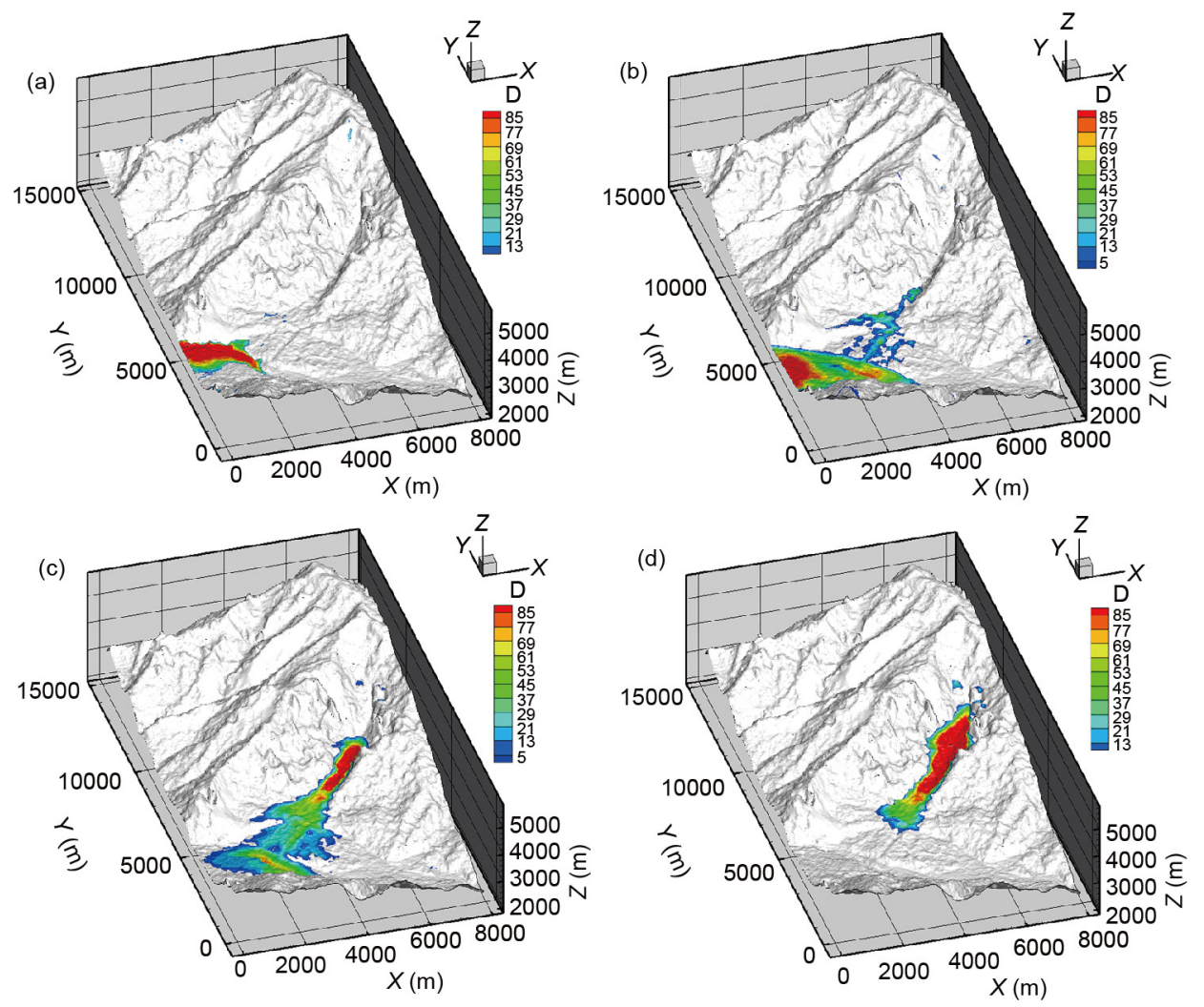

Figure 8 (Color online) Deposition patterns of calibrated cases with different bed friction angles. (a)-(d) Correspond the bed friction of $5^{\circ}, 10^{\circ}, 15^{\circ}$, and $20^{\circ}$, respectively. Depth distribution in meters is represented by different color shown in the scaleplate.

evolution of depth at the four positions is shown in Figure 10. Figure 11 plots the final deposit distribution.

From Figure 10, the leading edge of the granular avalanche reaches the Yigong River in about $1 \mathrm{~min}$ and continues to accumulate for another $1 \mathrm{~min}$, then the main avalanche body arrives with considerable momentum. Finally, the depth accumulates as high as $100 \mathrm{~m}$ and some of the material moves forward, leaving other granular material deposited to form a dammed lake. The duration from start to end is approximately $3 \mathrm{~min}$. The final avalanche forms a loudspeaker-shaped deposit with the upper side at the gorge and the bottom in the Yigong River, as shown in Figure 11.

In the simulation, the time duration from initiation to final stop is shown in both Figures 9 and 10. The widest de- posit spans about $3.2 \mathrm{~km}$ along the Yigong River channel, and spreads about $6 \mathrm{~km}$ from the gorge to the river as shown in Figure 11. The site survey $[2,3,51]$ shows that the final deposit of the event lasting around 3 min was also a loudspeaker-shaped bulk with a 3-km wide front in the Yigong River and about $4.6 \mathrm{~km}$ long from the front to the gorge, as for example seen in Figure 7 in ref. [51]. Furthermore, the simulated depths in Yigong River at Pos 1, Pos 2 and Pos 3 are between 60 and $90 \mathrm{~m}$, close to the actual depth of 50-80 m [51]. In summary, the simulated total duration, runout distance, and final deposit distribution of the avalanche event coincide fairly well with the field observations.

However, some deviations are evident in the simulation. The front of the numerical result seems to be more diverse 



Figure 9 (Color online) Simulated overviews of depth evolution (in meters) of Yigong avalanche at different moments. (a)-(h) Correspond to $0 \mathrm{~s}, 32 \mathrm{~s}, 42 \mathrm{~s}, 60 \mathrm{~s}, 114 \mathrm{~s}, 154 \mathrm{~s}, 183 \mathrm{~s}$, and $205 \mathrm{~s}$, respectively.

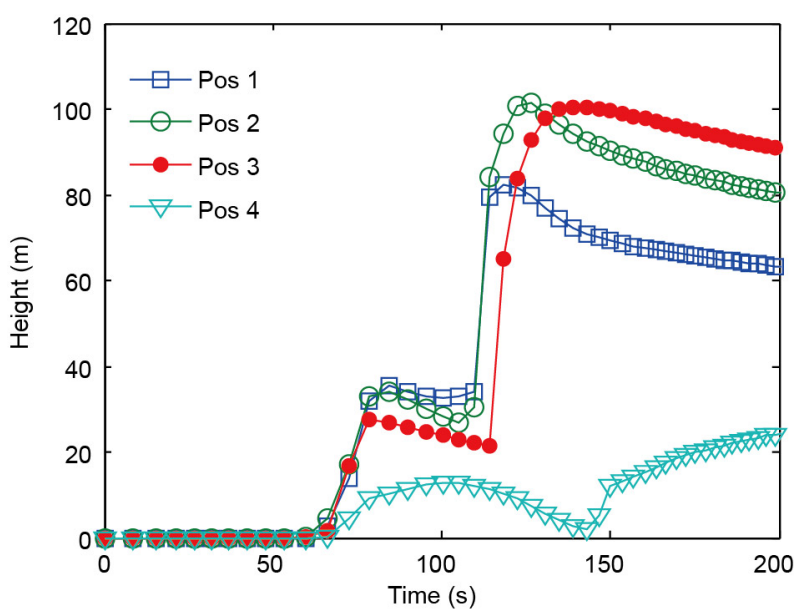

Figure 10 (Color online) Simulated depth evolution at 4 monitoring positions in Yigong River. The first arrival of the avalanche is about 1 min after failure, and the main body of the avalanche arrives after another 1 min.

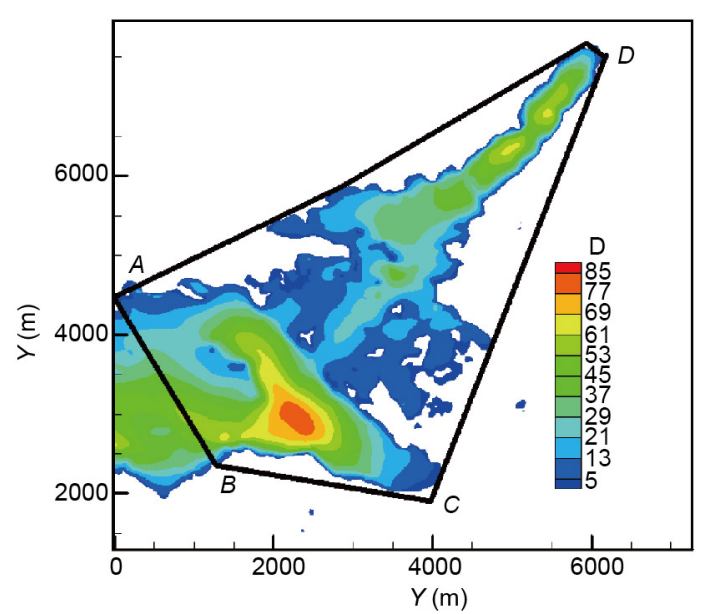

Figure 11 (Color online) Simulated final deposit distribution in meters. $D$ denotes the position of the gorge, and the formed dam across the Yigong River mainly lies in region $A B C$. 
than field observation, and the deposit area on the left Yigong River bank is narrower than reality. There are two main explanations for these discrepancies. First, the eroded material is deposited at a higher position in the numerical simulation than in reality. Second, the role of water gives rise to excess hydraulic pore pressure to accelerate the landslide along the slope, while pore pressure dissipation in the runout zone slows down the landslide. These phenomena, however, are ignored in the SH model. So the physical mechanism of flowing granular materials, either in bulk or at the bed interface, needs further improvements for better prediction. However, the simulations based on the SH model have already been shown to provide valuable information for hazard forecasting and mitigation.

\section{Conclusion}

Based on the Savage-Hutter model and the high resolution algorithm in computational gas dynamics with novel treatments of source terms and interface problem, a software platform is established to simulate both indoor experimental and field scale granular avalanches. The new solver is verified and validated by comparison of simulated two-dimensional avalanches of granular materials with experiments.

Systematic study on the roles of the two parameters in $\mathrm{SH}$ model shows that the bed friction governs runout distance while the internal friction mainly controls deposit spread extent. We find that runout efficiency is enhanced by reducing bed friction angle but is nearly independent of internal friction angle. The deposit distribution is wider for smaller earth pressure coefficients, which may result from a larger internal friction angle.

As a case study, the giant Yigong avalanche is well simulated, with results generally comparable to the field observations of material transport and deposit during the event. The Yigong avalanche lasts about $3 \mathrm{~min}$ for both observation and simulation, and the calculated final distribution shape, scale, and average depth of the deposit essentially agree with the site survey. Thus, it can be concluded that the new solver is generally capable of simulating both indoor experimental scale and geological scale granular avalanche. The runout analysis of giant granular avalanches may provide useful information regarding deposition behavior for hazard forecasting and mitigation in mountainous regions.

However, some deficiencies remain for field scale avalanche prediction. Consequently, the constitutive law and entrainment behavior of flowing granular materials require further exploration. Other issues such as fluidization, liquefaction, and size segregation along with their computer implementation are more challenging, and must be explored for future improved simulation of granular avalanche phenomena.
This work was supported by the National Natural Science Foundation of China (Grant Nos. 11602278, and 11432015), the Strategic Priority Research Program of Chinese Academy of Sciences (Grant No. XDB22040203), and the LMFS Foundation for Young Scientists, and the authors are grateful for the suggestions of anonymous reviewers.

1 R. Q. Huang, Geohazard Assessment of the Wenchuan Earthquake (in Chinese) (Science Press, Beijing, 2009), pp. 1-5.

2 Y. P. Ying, Hydrogeol. Eng. Geol. 27, 8 (2000).

3 M. J. Hu, Q. G. Cheng, and F. W. Wang, Chin. J. Rock Mech. Eng. 28, 138 (2009).

4 B. Andreotti, Y. Forterre, and O. Pouliquen, Granular Media: Between Fluid and Solid (Cambridge University Press, Cambridge, 2013), pp. 3-7.

5 Q. C. Sun, M. Y. Hou, and F. Jin, Physics and Mechanics of Granular Matter (Science Press, Beijing, 2011), pp. 242-265.

6 C. Ancey, J. Non-Newton. Fluid Mech. 142, 4 (2007).

7 Y. An, Q. Wu, C. Shi, and Q. Liu, Géotechnique 66, 670 (2016)

8 S. P. Pudasaini, Y. Wang, and K. Hutter, Nat. Hazards Earth Syst. Sci. 5, 799 (2005).

9 R. M. Iverson, and R. P. Denlinger, J. Geophys. Res. 106, 537 (2001).

10 M. Pastor, T. Blanc, B. Haddad, V. Drempetic, M. S. Morles, P. Dutto, M. M. Stickle, P. Mira, and J. A. F. Merodo, Arch. Computat. Methods Eng. 22, 67 (2015).

11 J. P. King, in Geotechnical Engineering: Meeting Society's Needs: Proceedings of 14th South East Asian Geotechnical Conference, edited by K. K. S. Ho, and K. S. Li (A. A. Balkema Publishers, Rotterdam, 2001), pp. 783-788.

12 S. B. Savage, and K. Hutter, J. Fluid Mech. 199, 177 (1989).

13 S. P. Pudasaini, and K. Hutter, J. Fluid Mech. 495, 193 (2003).

14 R. M. Iverson, and D. L. George, Proc. R. Soc. A-Math. Phys. Eng. Sci. 470, 20130819 (2014).

15 E. B. Pitman, and L. Le, Philos. Trans. R. Soc. A-Math. Phys. Eng. Sci. 363, 1573 (2005).

16 S. P. Pudasaini, J. Geophys. Res. 117, F03010 (2012).

17 F. P. Gao, J. H. Li, W. G. Qi, and C. Hu, Sci. China-Phys. Mech. Astron. 58, 124701 (2015).

18 G. Midi, Eur. Phys. J. E 14, 341 (2004).

19 X. L. Wang, and J. C. Li, Sci. China-Phys. Mech. Astron. 57, 2297 (2014).

20 X. Wang, and J. Li, Powder Tech. 275, 121 (2015)

21 J. M. N. T. Gray, and A. N. Edwards, J. Fluid Mech. 755, 503 (2014).

22 J. M. N. T. Gray, and V. A. Chugunov, J. Fluid Mech. 569, 365 (2006).

23 A. N. Edwards, and J. M. N. T. Gray, J. Fluid Mech. 762, 35 (2015).

24 S. McDougall, and O. Hungr, Can. Geotech. J. 42, 1437 (2005).

25 R. M. Iverson, and C. Ouyang, Rev. Geophys. 53, 27 (2015).

26 S. B. Savage, and K. Hutter, Acta Mech. 86, 201 (1991).

27 E. F. Toro, Riemann Solvers and Numerical Methods for Fluid Dynamics: A Practical Introduction (Springer, Berlin Heidelberg, 2013), pp. 493-503.

28 E. B. Pitman, C. C. Nichita, A. Patra, A. Bauer, M. Sheridan, and M. Bursik, Phys. Fluids 15, 3638 (2003).

29 S. F. Davis, SIAM J. Sci. Stat. Comput. 9, 445 (1988).

30 J. Zhai, L. Yuan, W. Liu, and X. Zhang, Int. J. Numer. Meth. Fluids 77, 381 (2015).

31 R. P. Denlinger, and R. M. Iverson, J. Geophys. Res. 106, 553 (2001).

32 D. L. George, and R. M. Iverson, Proc. R. Soc. A-Math. Phys. Eng. Sci. 470, 20130820 (2014).

33 M. Pelanti, F. Bouchut, and A. Mangeney, ESAIM-M2AN 42, 851 (2008).

34 M. Pelanti, F. Bouchut, and A. Mangeney, J. Comp. Phys. 230, 515 (2011).

35 M. Greco, M. Iervolino, A. Leopardi, and A. Vacca, Int. J. Sediment Res. 27, 409 (2012).

36 S. He, W. Liu, C. Ouyang, and X. Li, Nat. Hazards Earth Syst. Sci. 
Discuss. 2, 2151 (2014).

37 B. Cockburn, G. E. Karniadakis, and C. W. Shu, Discontinuous Galerkin Methods (Springer, Berlin Heidelberg, 2000), pp. 3-50.

38 S. Andersen, and L. Andersen, Comput. Geosci. 14, 137 (2010).

39 P. Jop, Y. Forterre, and O. Pouliquen, Nature 441, 727 (2006).

40 F. da Cruz, S. Emam, M. Prochnow, J. N. Roux, and F. Chevoir, Phys. Rev. E 72, 021309 (2005).

41 Y. Forterre, and O. Pouliquen, Annu. Rev. Fluid Mech. 40, 1 (2008).

42 P. Y. Lagrée, L. Staron, and S. Popinet, J. Fluid Mech. 686, 378 (2011).

43 A. Bermudez, and M. E. Vazquez, Comp. Fluids 23, 1049 (1994).

44 S. F. Bradford, and B. F. Sanders, J. Hydraul. Eng. 128, 289 (2002).
45 P. A. Sleigh, P. H. Gaskell, M. Berzins, and N. G. Wright, Comp. Fluids 27, 479 (1998).

46 H. J. Melosh, J. Geophys. Res. 84, 7513 (1979).

47 R. L. Shreve, Science 154, 1639 (1966).

48 R. M. Iverson, and R. G. LaHusen, Science 246, 796 (1989).

49 X. L. Wang, and J. C. Li, J. Eng. Geol. 24, 717 (2016).

50 T. Koch, R. Greve, and K. Hutter, Proc. R. Soc. A-Math. Phys. Eng. Sci. 445, 415 (1994).

51 J. Zhou, P. Cui, and M. Hao, Landslides 13, 39 (2016).

52 A. Lucas, A. Mangeney, and J. P. Ampuero, Nat. Commun. 5, 3417 (2014). 